\title{
Bis zu $40 \%$ aller Patienten mit leichten kognitiven Störungen sind depressiv
}

Fragestellung: Die hier vorliegende Studie wurde mit dem Ziel konzipiert, die Prävalenz depressiver Erkrankungen bei Patienten mit leichten kognitiven Einschränkungen (Mild Cognitive Impairment, MCI) zu bestimmen, zumal Depressionen offenbar ein Prädiktor für eine spätere dementive Entwicklung darstellen.

Hintergrund: Bisher existierende und weniger umfangreiche Untersuchungen haben bezüglich der oben aufgeführten Fragestellung sehr heterogene und inkonsistente Ergebnisse gebracht.

Patienten und Methodik: Die Datenbanken Medline, Embase und PsycINFO wurden im Hinblick auf bisherige Untersuchungen zu dieser Thematik gescreent. Eingeschlossen wurden ausschließlich solche Studien, in denen die primäre Stichprobe auf der Grundlage eines gesicherten MCI gebildet wurde und in denen depressive Symptome

Ismail Z, Elbayoumi H, Fischer CE et al. Prevalence of depression in patients with mild cognitive impairment. A systematic review and meta-analysis. JAMA Psychiatry 2017; 74: 58-67 oder die Diagnose einer Depression mit validierten psychometrischen Instrumenten erfasst wurden. Im Rahmen der statistischen Auswertung fand eine Stratifizierung im Hinblick auf mögliche deter- minierende Faktoren statt. Hierzu zählten zum Beispiel Patientenstichprobe, Art der Diagnostik und Durchschnittsalter.

Ergebnisse: Insgesamt konnten 57 Studien (20.892 Patienten) aufgrund der definierten Einschlusskriterien eingeschlossen werden. Bei $32 \%$ aller Patienten mit MCI fand sich eine komorbide depressive Erkrankung (95\%-Konfidenzintervall 27 - 37), allerdings waren die Ergebnisse auch trotz der engen Einschlusskriterien immer noch sehr heterogen $\left(I^{2}=90,7 \%\right)$. Untersuchungen auf der Grundlage von Bevölkerungsstichproben ergaben mit $25 \%$ eine niedrigere Prävalenz depressiver Erkrankungen bei MCI. Stichproben, die über eine Klinik oder eine Ambulanz erhoben wurden, hatten mit $40 \%$ eine signifikant höhere Prävalenz depressiver Erkrankungen. Die Art der Diagnostik (diagnostisches Interview durch einen Kliniker oder Self-Report) hatte keinen signifikanten Einfluss auf die Ergebnisse.

Schlussfolgerung: Patienten mit einer leichten kognitiven Störung leiden in einem hohen Prozentsatz auch unter einer behandlungsbedürftigen depressiven Erkrankung.

\section{- Kommentar von Andreas Broocks, Schwerin}

\section{Bei $\mathrm{MCl}$ komorbide Depressionen erkennen und behandeln}

Bei der vorliegenden Untersuchung handelt es sich um das bisher umfangreichste systematische Review mit Metaanalyse zu dieser Thematik. Eingeschlossen wurden 28 Studien aus Bevölkerungstichproben und 29 klinische Stichproben. Die Ergebnisse zeigen, dass bei Patienten, die unter einer milden kognitiven Beeinträchtigung leiden, unbedingt systematisch nach einer komorbiden depressiven Erkrankung gefahndet werden muss. Dies ist von hoher klinischer Relevanz, weil eine effektive Behandlung der Depression durchaus zu einer guten Besserung von kognitiven Beeinträchtigungen führen kann und weil im umgekehrten Falle eine chronifizierte depressive Erkrankung offenbar den Übergang in eine Demenz-Erkrankung begünstigen kann. Auch subsyndromale depressive Symptome können zu einer erheblichen Einschränkung der Lebensqualität von MCl-Patienten führen. Das weitergehende Ziel der Studie, nämlich die bisherige große Heterogenität zwischen den einzelnen Studien zu erklären, konnte nur zum Teil erreicht werden. Ein Gendereffekt konnte mangels Daten nicht untersucht werden, die Hypothese, dass das durchschnittliche Alter der Studienpopulation hier einen entscheidenden Einfluss ausübt, konnte nicht bestätigt werden. Die Ergebnisse waren auch unabhängig von dem diagnostischen Prozedere, das im Hinblick auf die Beurteilung der depressiven und der $\mathrm{MCl}$-Symptomatik verwendet wurde. Lediglich die Art der Stichprobe scheint bezüglich der Heterogenität einen signifikanten Einfluss auszuüben: $40 \%$ aller Patienten, bei denen in einem klinischen Setting eine milde kognitive Beeinträchtigung diagnostiziert wurde, leiden unter einer klinisch relevanten depressiven Symptomatik - im Vergleich zu $25 \%$ in Bevölkerungsstichproben!

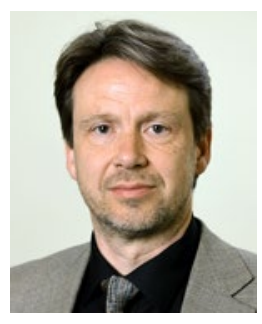

Prof. Dr. med. Andreas Broocks, Schwerin

Carl-Friedrich-Flemming-Klinik

HELIOS Kliniken Schwerin

E-Mail: andreas.broocks@helios-kliniken.de 